\title{
The olfactory bulb modulates entorhinal cortex oscillations during spatial working memory
}

\author{
Morteza Salimi ${ }^{1}$, Farhad Tabasi ${ }^{1}, 2$, Milad Nazari ${ }^{3}$, Sepideh Ghazvineh ${ }^{1}$, Alireza Salimi ${ }^{4}$, Hamidreza Jamaati ${ }^{4}$ and \\ Mohammad Reza Raoufy ${ }^{1,2^{*}}$ (1)
}

\begin{abstract}
Cognitive functions such as working memory require integrated activity among different brain regions. Notably, entorhinal cortex (EC) activity is associated with the successful working memory task. Olfactory bulb (OB) oscillations are known as rhythms that modulate rhythmic activity in widespread brain regions during cognitive tasks. Since the $\mathrm{OB}$ is structurally connected to the $\mathrm{EC}$, we hypothesized that $\mathrm{OB}$ could modulate $\mathrm{EC}$ activity during working memory performance. Herein, we explored $\mathrm{OB}-\mathrm{EC}$ functional connectivity during spatial working memory performance by simultaneous recording local field potentials when rats performed a Y-maze task. Our results showed that the coherence of delta, theta, and gamma-band oscillations between OB and EC was increased during correct trials compared to wrong trials. Cross-frequency coupling analyses revealed that the modulatory effect of OBs low-frequency phase on EC gamma power and phase was enhanced when animals correctly performed working memory task. The influx of information from OB to EC was also increased at delta and gamma bands within correct trials. These findings indicated that the modulatory influence of OB rhythms on EC oscillations might be necessary for successful working memory performance.
\end{abstract}

Keywords: Olfactory bulb, Entorhinal cortex, Working memory, Functional connectivity

\section{Background}

Working memory is a workspace for temporary storage and active manipulating information for ongoing decision-making [1-3]. Successful working memory performance depends on organized communication between brain regions [1-3]. The entorhinal cortex (EC) is a part of the medial temporal lobe system, positioned as a 'gateway' between neocortical areas and the hippocampal memory system [4]. It has been shown that EC is acting as a temporal buffer of incoming information to the hippocampus [5]. Lesioning studies also exhibited that

\footnotetext{
*Correspondence: raoufy@modares.ac.ir

${ }^{1}$ Department of Physiology, Faculty of Medical Sciences, Tarbiat Modares University, Tehran, Iran
}

Full list of author information is available at the end of the article
EC ablation disrupts the working memory performance [6-8]. Hence, EC is likely pivotal for successful working memory performance [9].

Entorhinal cortex is closely connected with the olfactory bulb (OB); sensory information directly reaches the $\mathrm{EC}$ from $\mathrm{OB}$ and bypasses the thalamus, unlike other sensory systems $[10,11]$. Apical dendrites in layer II/III pyramidal and stellate in the lateral portion of EC cells receive projections from OB's mitral cells $[12,13]$. On the other hand, $\mathrm{OB}$ oscillations and their coupling with other brain regions are correlated with cognitive functions $[14,15]$. Removing or lesioning the $\mathrm{OB}$ leads to cognitive impairments such as attentional tasks, reference memory, delayed matching, reversal memory, and working memory deficits [16-18]. It has been indicated that low-gamma oscillations of $\mathrm{OB}$ are prominent during

(c) The Author(s) 2021. This article is licensed under a Creative Commons Attribution 4.0 International License, which permits use, sharing, adaptation, distribution and reproduction in any medium or format, as long as you give appropriate credit to the original author(s) and the source, provide a link to the Creative Commons licence, and indicate if changes were made. The images or other third party material in this article are included in the article's Creative Commons licence, unless indicated otherwise in a credit line to the material. If material is not included in the article's Creative Commons licence and your intended use is not permitted by statutory regulation or exceeds the permitted use, you will need to obtain permission directly from the copyright holder. To view a copy of this licence, visit http://creativeco mmons.org/licenses/by/4.0/. 
conscious state and have an essential role in synchronizing neural circuits related to spatial declarative memory $[19,20]$. However, the high-gamma oscillations of OB are more periodic during spontaneous exploration in a new environment [20]. Since the activity of OB and EC contributes to memory function, it seems that interaction between $\mathrm{OB}$ and $\mathrm{EC}$ oscillations may play a role in the successful working memory performance. To date, no study is available to assess functional connectivity between $\mathrm{OB}$ and $\mathrm{EC}$ during the working memory performance.

Electrophysiological investigations have demonstrated that the functional connectivity within brain regions can be essential for cognitive behaviors [21]. Oscillatory coupling between disparate brain areas facilitates interregional communication, essential for information processing during a behavior [22]. Specifically, cross-frequency coupling (CFC) is considered the optimal coding supporting information transfer during a cognitive performance, including working memory $[22,23]$. Therefore, the strength of functional coupling between brain regions may represent a brain code of memory circuit activation.

Altogether, we hypothesized that $\mathrm{OB}$ oscillations coordinate the activity of EC, which mediates the working memory performance. We investigated the OB-EC functional connectivity during the working memory task by simultaneous recording local field potentials (LFPs) when rats spontaneously alternated in the Y-maze. The OB-EC circuit coherence was computed to evaluate the frequency-specific functional connectivity when rats performed the working memory task. Then, we addressed the modulatory effect of the OB low-frequency phase on EC high frequencies phase and power using CFC analyses. Finally, the influx of information from $\mathrm{OB}$ to $\mathrm{EC}$ is measured with Granger causality.

\section{Methods}

This experiment was performed on pathogen-free male Wistar rats weighing 210-230 g (9-10 weeks) obtained from the Tarbiat Modares University animal house (Tehran, Iran). Rats were kept in standard animal research facilities, in which food and water were available. For electrode implantation, anesthesia was induced using ketamine/xylazine $(100 \mathrm{mg} / \mathrm{kg}$ and $10 \mathrm{mg} / \mathrm{kg}$, respectively), and the head was fixed. Next, two stainless-steel recording electrodes $(127 \mu \mathrm{m}$ in diameter, A.M. system Inc., USA) implanted into the OB (AP: $8.5 \mathrm{~mm}$, ML: $-1 \mathrm{~mm}, \mathrm{DV}:-1.5 \mathrm{~mm}$ ), and lateral portion of EC (AP: $-7.04 \mathrm{~mm}$; L: $-5.5 \mathrm{~mm}$; DV: $-6.5 \mathrm{~mm}$ ). To confirm the electrode location, the lesion in place of the electrode was done, the animal's brain was fixed with $4 \%$ paraformaldehyde, and a $200 \mu \mathrm{m}$ coronal section of the brain was prepared. The brain slices were compared to the matching slices visually in the rat brain atlas (Fig. 1b) [24].

After 2 weeks of recovery from surgery, for recording LFP signals, a head socket was fixed on the animals' heads, which was connected to the miniature buffer head stage with high-input impedance (BIODAC-A, TRITA Health Tec., Tehran, Iran). The signals were amplified (1000 amplification gain), digitized at $1 \mathrm{kHz}$ and lowpass filtered $<250 \mathrm{~Hz}$ via $\mathrm{AC}$ coupled with the recording

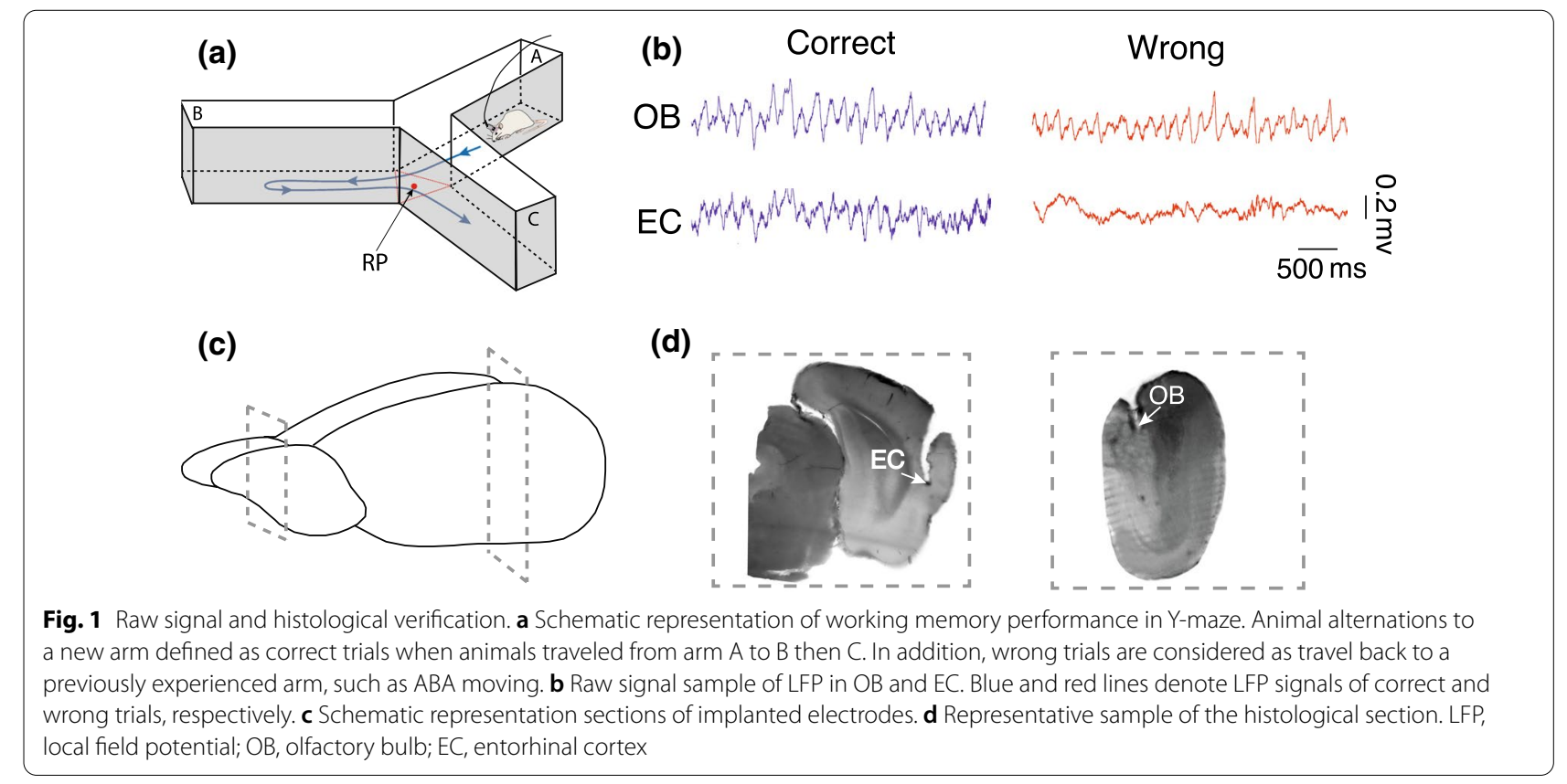


system (BIODAC-ESR18622, TRITA Health Technology Co., Tehran, Iran). Then, we recorded LFP signals simultaneously from $\mathrm{OB}$ and $\mathrm{EC}$ of rats spontaneously alternating in the Y-shaped maze. The signals processed offline using custom-written MATLAB software (Mathworks Inc., USA).

A Y-shaped black Plexiglas maze with $50 \mathrm{~cm}$ length, $10 \mathrm{~cm}$ width, and $25 \mathrm{~cm}$ height were chosen for the spatial working memory task. Animals were habituated for 2 consecutive days in the maze. Next, the animals were individually placed in the center of one arm, designated hereafter as arm A, allowing them to explore for $10 \mathrm{~min}$ freely. Spontaneous alternations were counted according to the previous protocol of working assessment [25]. Briefly, the correct performance was defined as animals' alternation to a new arm (i.e., visit from A to B or C, which are designated to the other arms, respectively). In addition, wrong trials are considered to travel back to a previously experienced arm, such as ABA moving. All movements were recorded with a ceiling-mounted video camera which was synchronized with LFP recording. Rats with misplaced electrodes or could not perform the task were excluded from the study. All procedures were in accordance with NIH Guidance for the Care and Use of Laboratory Animals (2011) and approved by the Ethics Committee of Faculty of Medical Sciences, Tarbiat Modares University (IR.MODARES.REC.1398.037).

\section{Signal processing}

We used a specific epoch of the signals recorded during spatial working memory performance in the Y-maze. Each epoch was selected $1 \mathrm{~s}$ before and during animal exploration of the maze center, both for correct and wrong trials. We identified delta $(<4 \mathrm{~Hz})$, theta $(4-12 \mathrm{~Hz})$, low-gamma $(30-50 \mathrm{~Hz})$, and high-gamma $(50-80 \mathrm{~Hz})$ via applying low- and bandpass filtering (Fig. 1a). To calculate OB and EC's coherence, computed magnitude-squared coherence by the mscohere function in MATLAB was used. Phase-power analysis was done by first obtaining the LFP phase by computing Hilbert's transform phase of the filtered signal in low frequencies (delta and theta) bands and gamma power calculated using the spectrogram with one sample time step. Then, we binned the low-frequency phases into 120 bins, each $3^{\circ}$ wide, and the average power of gamma samples calculated in each bin. Coupling strength was expressed as the resultant length vector, which was the average of power vectors in the low-frequency phases. Finally, to soften, we used the Gaussian kernel with a standard deviation of 10 and a size of 100 [26, 27].

Phase-phase coupling was calculated according to our recent study [28]. Briefly, we compute a matrix whose element $(i, j)$ represents the ratio of the total number in time samples that the $\mathrm{OB}$ phase at low-frequency (delta and theta) oscillations are placed at the angular bin number $i$ simultaneous with a phase of EC high frequencies (gamma 1 and 2) in the angular bin of the number $j$. To evaluate the influx of information from $\mathrm{OB}$ to $\mathrm{EC}$, we measured the Granger causality index. This analysis is based on the idea that oscillations in one brain region help predict others [29]. In this regard, we applied the Partial Directed Coherence (PDC) method, presumed that the past knowledge of the "cause" improved the prediction of the present state of the "effect," in contrast to prediction, which uses only the effect's past. We first examined the stationarity of all around the signals and estimated the autoregressive model order $(p=100)$ using Akaike's information criterion to calculate PDC. Next, by calculating the coefficients of the autoregressive model, we transferred the coefficients into the frequency domain using discrete Fourier transform [30-35].

\section{Statistical analysis}

GraphPad Prism (GraphPad Software, San Diego, CA) was used for statistical analyses. The data distribution normality was assessed with the Kolmogorov-Smirnov test within each parameter. The paired $t$ test was used for data comparison. The statistical significance was defined as levels less than 0.05 .

\section{Results}

$\mathrm{OB}$ oscillations are coherent with EC during correct spatial working memory performance trials

We simultaneously recorded LFP from OB and EC during spontaneous alternating in the Y-maze to study the OB-EC circuit connectivity in the spatial working memory performance. We observed that the OB-EC coherence in the delta and theta range during correct trials was significantly higher than wrong trials $(p<0.05$; Fig. $2 \mathrm{a}-\mathrm{c})$. In addition, the coherence of gamma $1(30-50 \mathrm{~Hz})$ and gamma $2(50-80 \mathrm{~Hz})$ oscillations of the OB-EC circuit was significantly higher during correct spatial working memory performance ( $p<0.05$, Fig. $2 \mathrm{~d}-\mathrm{f})$. Therefore, the enhanced coherence at both low and high frequencies in the OB-EC circuit is associated with correct spatial working memory performance.

\section{The $\mathrm{OB}$ phase strongly modulates $\mathrm{EC}$ power and phase during correct working memory performance}

We observed that the phase of both delta and theta oscillations in $\mathrm{OB}$ strongly modulates gamma's power in $\mathrm{EC}$ during correct trials (Fig. 3). The coupling of OB low-frequency phases with EC gamma-2 phases was significantly increased in correct compared to wrong trials (Fig. 4). However, no noticeable changes were found in OB-EC delta or theta gamma-1 phase locking during correct 


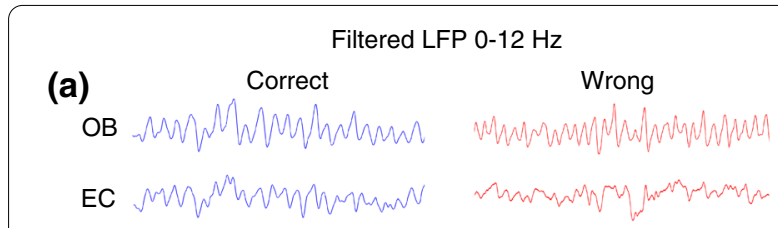

(b)
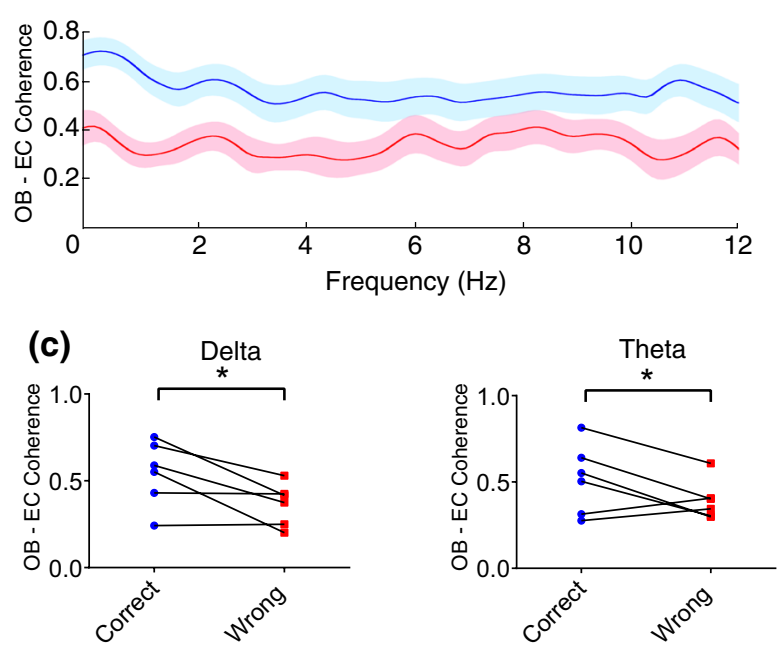

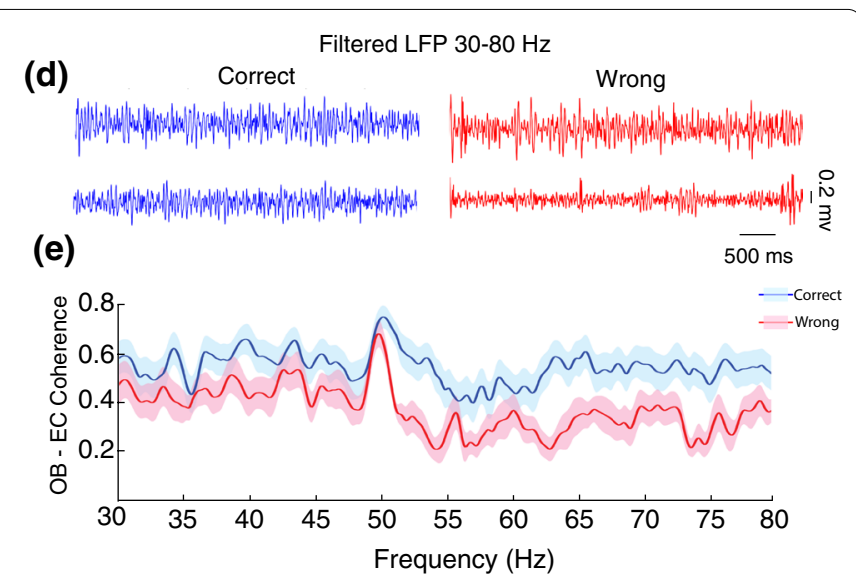

(e)

(f)

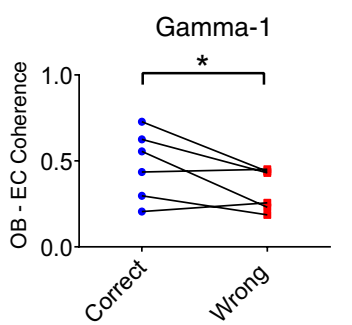

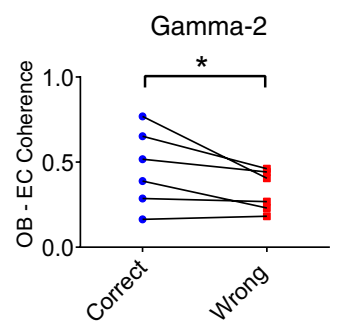

Fig. 2 Increased coherence between $O B$ and EC during correct trials. a, d Representative samples of filtered LFP signals in OB and EC at low $(0-12 \mathrm{~Hz})$ and high frequencies $(30-80 \mathrm{~Hz})$, respectively, during correct (blue) and wrong (red) trials. $\mathbf{b}, \mathbf{e} \mathrm{OB}-\mathrm{EC}$ coherence across the delta $(<4 \mathrm{~Hz})$, theta $(4-12 \mathrm{~Hz})$, gamma-1 $(30-50 \mathrm{~Hz})$, and gamma-2 $(50-80 \mathrm{~Hz})$ frequency bands. $\mathbf{c}$, f Coherence between OB and EC was significantly higher within correct than wrong trials (data averaged for either correct and wrong trials of each animal completed the task $2 \mathrm{~s}$ pre and $1 \mathrm{~s}$ post the RP). Plots show the comparisons between the coherence mean of correct (blue dots) and wrong (red dots) trials of each animal (connected with lines). Data were analyzed by paired $t$ test, $n=6 .{ }^{*} p<0.05,{ }^{* *} p<0.01$ and ${ }^{* * *} p<0.001$. RP, reference point; LFP, local field potential; OB, olfactory bulb; EC, entorhinal cortex

vs. wrong trials. Overall, this analysis revealed that EC gamma oscillations depend on the phase transition of $\mathrm{OB}$ signals when animals correctly performed the working memory task.

\section{The influx of information from $O B$ to $E C$ increased during correct working memory performance}

We additionally investigated whether EC oscillations are causally related to OB signals, while animals performed the spatial working memory task. Granger causality analysis revealed that the influx of information from $\mathrm{OB}$ to EC at gamma oscillations increased when rats made the correct performance within working memory task (Fig. 5). These results support the idea that OB not only modulatory but also has a casual effect on EC during correct working memory performance.

\section{Discussion}

Our results revealed that OB-EC coherence increased in the delta, theta, and gamma bands during correct spatial working memory task trials. Moreover, the phase of OB low frequencies had a more modulatory effect on the EC gamma power and phase during successful working memory performance. In addition, the influx of information from $\mathrm{OB}$ to EC was enhanced when animals correctly performed the working memory task.

Coherent activity is a hallmark of neuronal networks during cognitive performance, which requires structural coupling of neurons [36]. Given that $\mathrm{OB}$ and EC have monosynaptic connections and their activities are extensively related to the working memory, we presumed that enhanced connectivity in the OB-EC circuit might represent correct performance. To test this hypothesis, we assessed the $\mathrm{OB}-\mathrm{EC}$ functional connectivity when rats were performing the Y-maze task. Our analysis exhibited that $\mathrm{OB}$ oscillations at both low- and high-frequency ranges are strongly coherent with EC activity when rats made the correct response. It has been reported that coherent oscillations at low-frequency ranges, including delta and theta, mediate the interaction between brain areas in large-scale cognitive tasks such as memory performance [37, 38]. Furthermore, coherent oscillations at high frequency have also been thought to link distributed neural assemblies, a function that is probably important for network processes [39-41]. Here, the coherence 


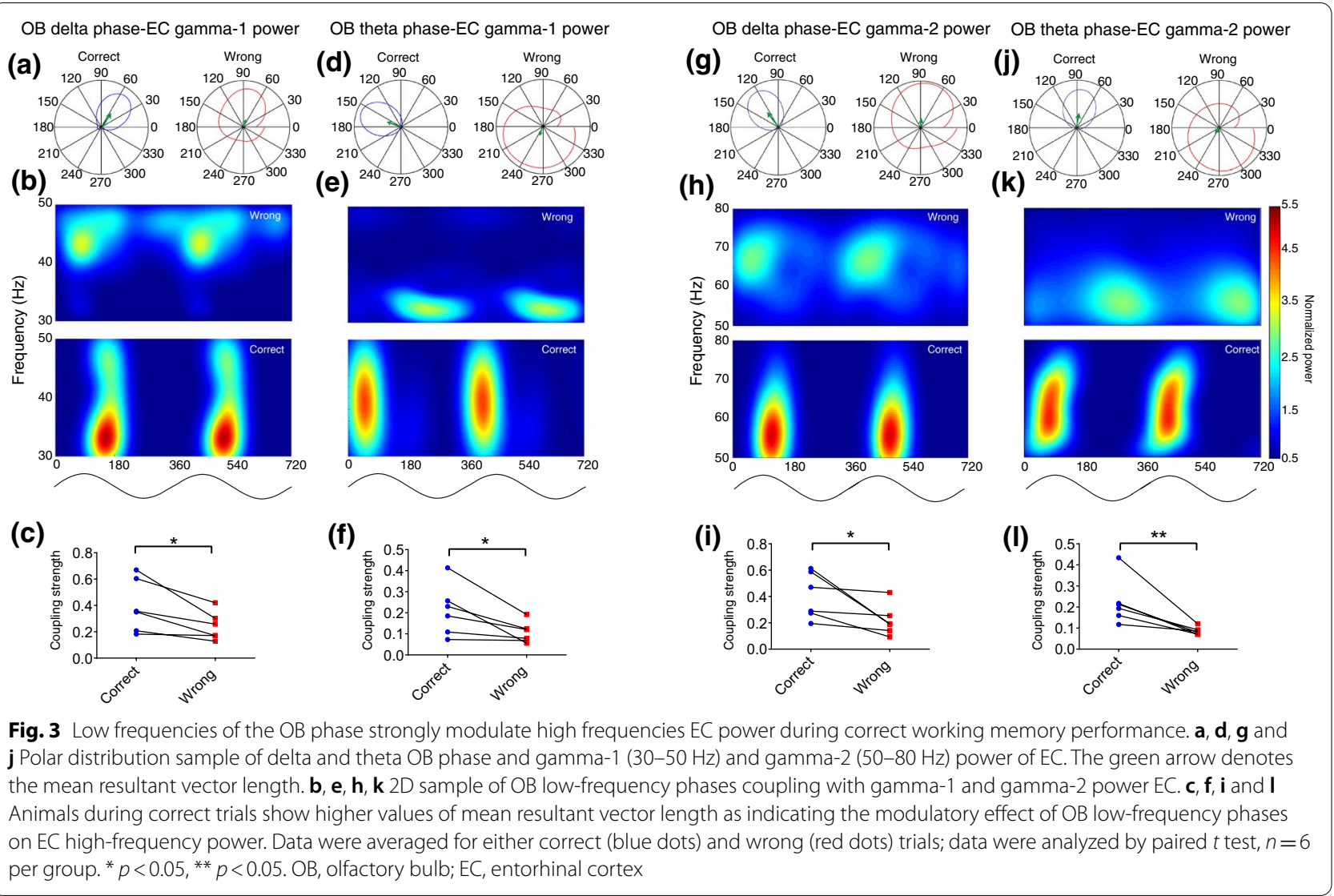

(a)

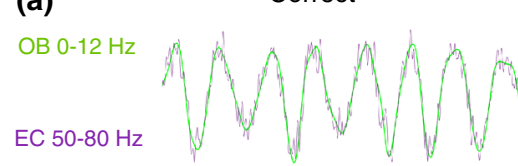

(b)

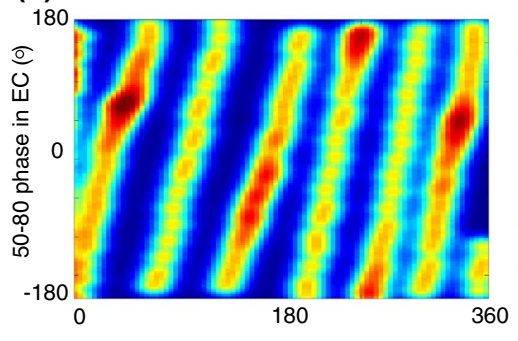

$0-12$ phase in $\mathrm{OB}\left({ }^{\circ}\right)$
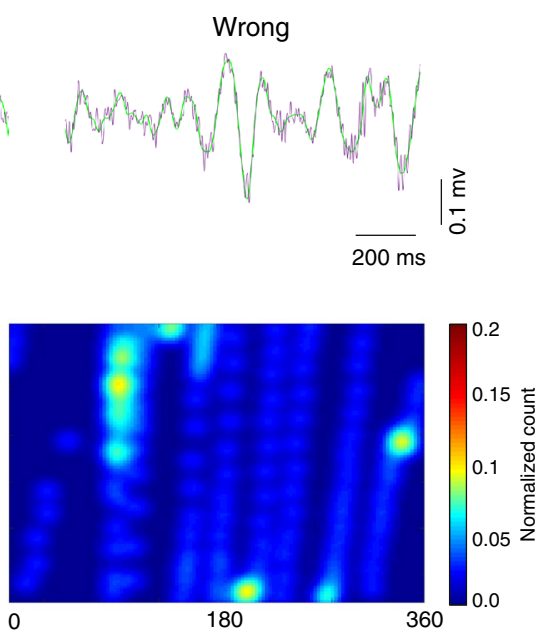

$0-12$ phase in $\mathrm{OB}\left({ }^{\circ}\right)$ (c)
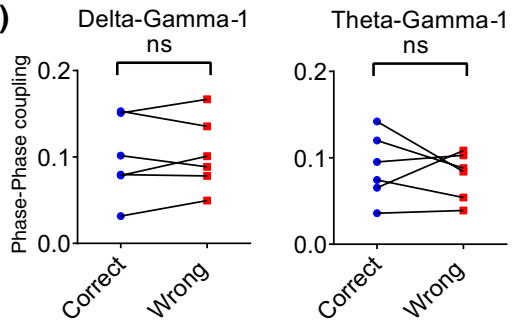

(d)

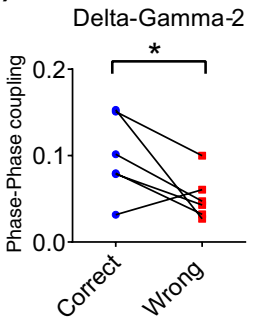

Theta-Gamma-2

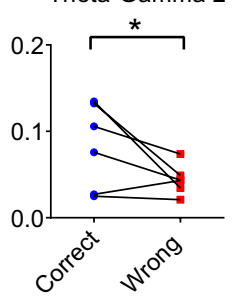

Fig. 4 OB phase increased coupling with the EC phase during correct working memory performance. a Representative sample filtered LFP that gamma-2 (50-80 Hz) superimposed on low frequencies (0-12) within correct (left) and wrong trials (right). b Sample of phase-phase 2D histogram of OB low frequencies (0-12) and EC gamma-2 during correct and wrong trials. c, $\mathbf{d}$ OB-EC circuit oscillations showed an increased phase-phase coupling at delta-gamma-2 and theta-gamma-2 during correct trials vs. wrong. Data were averaged for either correct (blue dots) and wrong (red dots), data were analyzed by paired $t$ test, $n=6$ per group. ${ }^{*} p<0.05$; ns, not significant. OB, olfactory bulb; EC, entorhinal cortex 


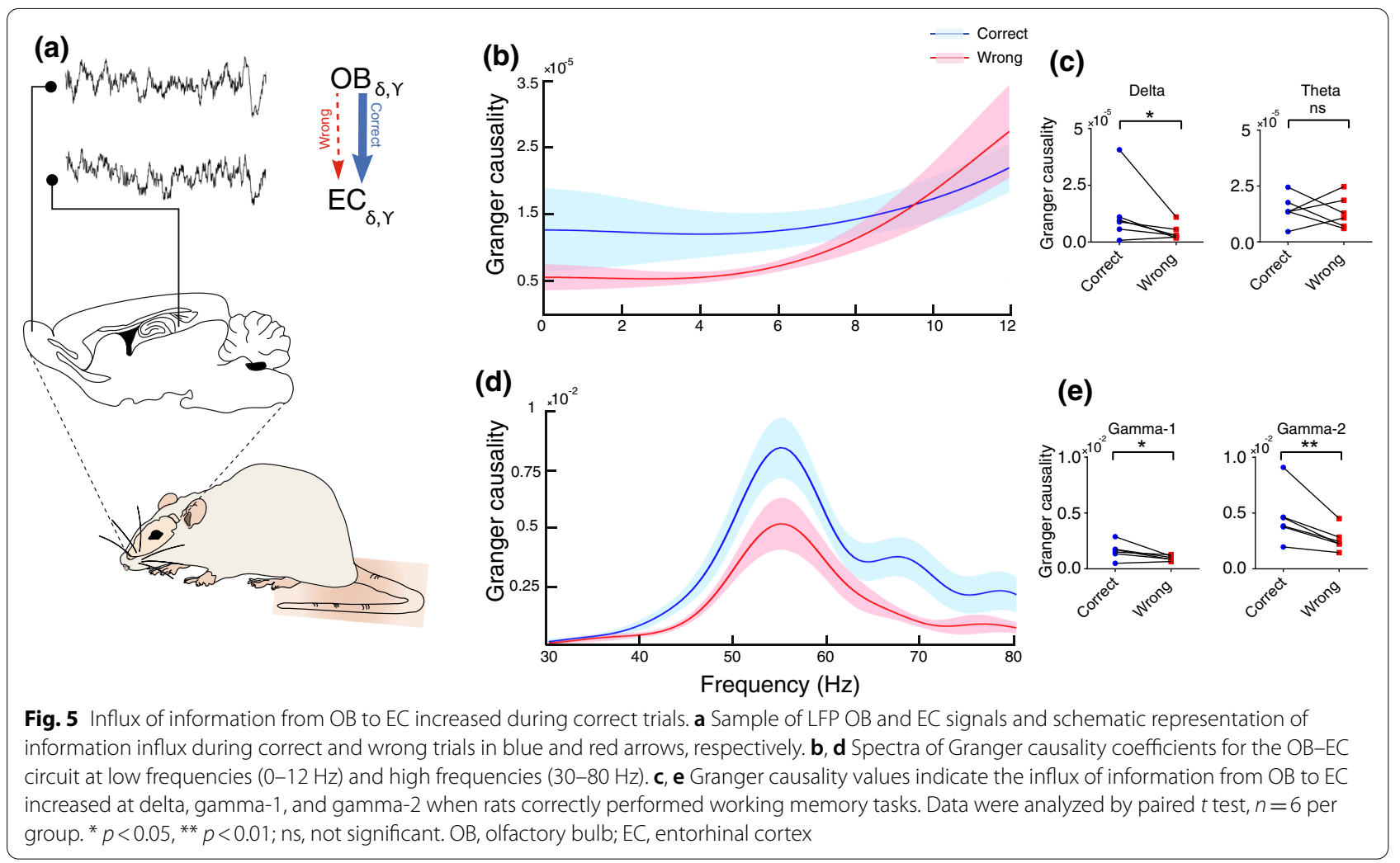

findings point to the close communication between $\mathrm{OB}$ and EC to promote working memory performance.

There is growing evidence demonstrating that $\mathrm{OB}$ activity entrains LFP oscillations in widespread regions of the rodent brain [42]. Hereafter our findings represented a strong coherence; we then ask whether correct working memory performance is coded by modulation of EC oscillations by OB activity. Interestingly, our OBEC phase-power coupling analysis results showed that delta-gamma and theta-gamma coupling are significantly higher during correct trials. Moreover, the $\mathrm{OB}$ delta and theta phases modulated the phase of either low and high gamma-band oscillations in EC during spatial working memory, and the value of such modulation increased during the correct performance. These findings are consistent with previous studies that suggested that CFC across the brain regions plays a functional role in neuronal computation, communication, and learning [43-45].

Importantly, it can be suggested that gamma oscillations in hippocampal formation, which contribute to memory performance, can be modulated by OB rhythm. It is previously reported that gamma oscillations in the hippocampus consist of two distinct frequency components (low and high-gamma). Precisely, high-gamma in the hippocampus raised from EC, low-gamma oscillations are thought to be generated from intrinsic sources $[46,47]$. Our result exhibited that oscillations in lateral $\mathrm{EC}$, which is reciprocally connected with $\mathrm{OB}$ via direct projections $[12,13]$ are driven from $\mathrm{OB}$ when animals performed the working memory for the first time. Therefore, $\mathrm{OB}$ oscillations through modulation of EC may affect the gamma activity in the hippocampus contributing to successful working memory performance. However, further studies are required to understand better OB's mechanisms in brain oscillations and behavioral performance.

Whereas high-frequency oscillations represent local cortical processing, low-frequency rhythms can dynamically entrain across distributed brain regions [47]. CFC constitutes a mechanism to transfer information within brain circuits operating at the behavioral performance [47]. These observations confirm our hypothesis that $\mathrm{OB}$ can coordinate EC during working memory performance.

An intriguing question is how OB drives these effects? The possible answer is an internal driver in OB generating and facilitating both local and long-distance brain rhythms. In mammals, OB expresses prominent neural oscillations $[48,49]$, which is shown to synchronize neural activities of the brain networks [50]. These oscillations are generated during nasal respiration by stimulating olfactory sensory neurons (OSNs) [51]. Olfactory neural 
oscillations that are phased-locked to respiration cycles, called respiration-entrained brain oscillations [12], reach remote areas $[52,53]$ and modulate rhythmic activity in non-olfactory regions [54]. Therefore, these rhythms synchronize OB oscillations with long-distance areas, especially during a cognitive performance [42]. For example, it has been reported that $\mathrm{OB}$ and hippocampus synchronized at theta oscillation during cognitive performances $[55,56]$.

Moreover, increased delta oscillations during a spatial working memory task in $\mathrm{MPFC}$, VTA, and hippocampus [57], were assumed to be entrained by respiration rhythm [42]. However, for the first time, the present work results exhibited that $\mathrm{OB}$ oscillations can modulate EC activities during working memory performance. Our explanation for these observations is that OSNs through nasal respiration may coordinate $\mathrm{OB}$ oscillations, and then OB drives the changes throughout distant areas, including EC. OSNs are shown to have dual functions that respond not only to the chemical stimulus (odor) but also are activated by nasal airflow [51]; therefore, it is likely that OB oscillations are generating with odor-free airflow via OSN mechanical stimulation [51, 58]. In addition to respiration, other sensory information input can also affect the connectivity in the OB-EC circuit $[59,60]$. For instance, experimental data have confirmed that the expression of odor-specific representations in the EC is required to guide navigation during successful associative memory performance [59]. Moreover, sniffing as a main sensory input during cognitive performance has also been associated with hippocampal oscillations during learning [55]. However, more studies are needed to uncover the underlying mechanism in which OB modulates EC during working memory performance.

$\mathrm{OB}$ oscillations have been previously shown to cause hippocampus rhythm [61]. Therefore, we further assumed that EC activity is coherent and modulated with $\mathrm{OB}$ oscillations, but EC oscillations are also causally related to OB activity. In the current study, the Granger causality calculation demonstrated that the increment in the influx of information from $\mathrm{OB}$ to $\mathrm{EC}$ is associated with correct working memory performance. This evidence, consistent with our other findings, supports a model in which the OB sends information to the EC that may be essential for successful performance during the working memory.

\section{Conclusions}

In summary, we found that $\mathrm{OB}$ oscillation modulates the rhythmic activity of EC in association with the correct performance of the spatial working memory task. These findings provide new insight into OB activity's effect on remote areas associated with cognition and behavior in rodents. However, further studies are needed to uncover how $\mathrm{OB}$ influences the brain regions during working memory performance.

\section{Acknowledgements \\ We would like to thank Mr. Reza Vaziri for his technical assistance.}

\section{Authors' contributions}

MS and MRR designed the research; MS performed experiments and data acquisition; MS and MN contributed to the data analysis; MS, FT, MRR contributed to the data interpretation; MS, FT, and SG wrote the manuscript; $A R, H J$, and MRR have supervised the study. All authors read and approved the final manuscript.

\section{Funding}

This study was supported by Tarbiat Modares University (Grant Number IG-39709). The funders had no role in the study.

\section{Availability of data and materials}

The data sets used and analyzed during the current study are available from the corresponding author on reasonable request.

\section{Declarations}

Ethics approval and consent to participate

All procedures were in accordance with NIH Guidance for the Care and Use of Laboratory Animals (2011) and approved by the "Ethics Committee of Faculty of Medical Sciences, Tarbiat Modares University [IR.MODARES.REC.1398.037]."

\section{Consent for publication}

Not applicable.

\section{Competing interests}

The authors declare that they have no competing interests.

\section{Author details}

${ }^{1}$ Department of Physiology, Faculty of Medical Sciences, Tarbiat Modares University, Tehran, Iran. ${ }^{2}$ Institute for Brain Sciences and Cognition, Faculty of Medical Sciences, Tarbiat Modares University, Tehran, Iran. ${ }^{3}$ Electrical Engineering Department, Sharif University of Technology, Tehran, Iran. ${ }^{4}$ Chronic Respiratory Diseases Research Center, National Research Institute of Tuberculosis and Lung Diseases, Shahid Beheshti University of Medical Sciences, Tehran, Iran.

Received: 25 April 2021 Accepted: 16 June 2021

Published online: 30 June 2021

\section{References}

1. Just MA, Carpenter PA (1992) A capacity theory of comprehension: individual differences in working memory. Psychol Rev 99(1):122

2. Fuster JM (1995) Memory in the cerebral cortex: an empirical approach to neural networks in the human and nonhuman primate. The MIT Press

3. Baddeley A (1992) Working memory. Science 255(5044):556-559

4. Eichenbaum H, Lipton PA (2008) Towards a functional organization of the medial temporal lobe memory system: role of the parahippocampal and medial entorhinal cortical areas. Hippocampus 18(12):1314-1324

5. Hasselmo ME, Fransen E, Dickson C, Alonso AA (2000) Computational modeling of entorhinal cortex. Ann N Y Acad Sci 911(1):418-446

6. Otto T, Eichenbaum H (1992) Complementary roles of the orbital prefrontal cortex and the perirhinal-entorhinal cortices in an odor-guided delayed-nonmatching-to-sample task. Behav Neurosci 106(5):762

7. Kopniczky Z, Dochnal R, Mácsai M, Pál Á, Kiss G, Mihály A, Szabó G (2006) Alterations of behavior and spatial learning after unilateral entorhinal ablation of rats. Life Sci 78(23):2683-2688

8. Buzsáki G, Moser El (2013) Memory, navigation and theta rhythm in the hippocampal-entorhinal system. Nat Neurosci 16(2):130-138 
9. Fyhn M, Molden S, Witter MP, Moser El, Moser M-B (2004) Spatial representation in the entorhinal cortex. Science 305(5688):1258-1264

10. Luskin MB, Price JL (1983) The topographic organization of associational fibers of the olfactory system in the rat, including centrifugal fibers to the olfactory bulb. J Comp Neurol 216(3):264-291

11. Igarashi KM, leki N, An M, Yamaguchi Y, Nagayama S, Kobayakawa K, Kobayakawa R, Tanifuji M, Sakano H, Chen WR (2012) Parallel mitral and tufted cell pathways route distinct odor information to different targets in the olfactory cortex. J Neurosci 32(23):7970-7985

12. Gretenkord S, Kostka JK, Hartung H, Watznauer K, Fleck D, Minier-Toribio A, Spehr M, Hanganu-Opatz IL (2019) Coordinated electrical activity in the olfactory bulb gates the oscillatory entrainment of entorhinal networks in neonatal mice. PLoS Biol 17(1):e2006994

13. Wouterlood F, Nederlof J (1983) Terminations of olfactory afferents on layer II and III neurons in the entorhinal area: degeneration-Golgi-electron microscopic study in the rat. Neurosci Lett 36(2):105-110

14. Zelano C, Jiang H, Zhou G, Arora N, Schuele S, Rosenow J, Gottfried JA (2016) Nasal respiration entrains human limbic oscillations and modulates cognitive function. J Neurosci 36(49):12448-12467

15. Salimi M, Ghazvineh $S$, Zare M, Parsazadegan T, Dehdar K, Nazari M, Mirnajafi-Zadeh J, Jamaati H, Raoufy MR (2019) Distraction of olfactory bulb-medial prefrontal cortex circuit may induce anxiety-like behavior in allergic rhinitis. PLoS ONE 14(9):e0221978

16. Hozumi S, Nakagawasai O, Tan-No K, Niijima F, Yamadera F, Murata A, Arai Y, Yasuhara H, Tadano T (2003) Characteristics of changes in cholinergic function and impairment of learning and memory-related behavior induced by olfactory bulbectomy. Behav Brain Res 138(1):9-15

17. van Rijzingen IM, Gispen WH, Spruijt BM (1995) Olfactory bulbectomy temporarily impairs morris maze performance: an ACTH (4-9) analog accellerates return of function. Physiol Behav 58(1):147-152

18. Yamamoto T, Jin J, Watanabe S (1997) Characteristics of memory dysfunction in olfactory bulbectomized rats and the effects of cholinergic drugs. Behav Brain Res 83(1-2):57-62

19. Kay LM (2003) Two species of gamma oscillations in the olfactory bulb: dependence on behavioral state and synaptic interactions. J Integr Neurosci 2(01):31-44

20. Zhong W, Ciatipis M, Wolfenstetter T, Jessberger J, Müller C, Ponsel S, Yanovsky Y, Brankačk J, Tort AB, Draguhn A (2017) Selective entrainment of gamma subbands by different slow network oscillations. Proc Natl Acad Sci 114(17):4519-4524

21. Gągol A, Magnuski M, Kroczek B, Kałamała P, Ociepka M, Santarnecchi E, Chuderski A (2018) Delta-gamma coupling as a potential neurophysiological mechanism of fluid intelligence. Intelligence 66:54-63

22. Fell J, Axmacher N (2011) The role of phase synchronization in memory processes. Nat Rev Neurosci 12(2):105-118

23. Jensen O, Colgin LL (2007) Cross-frequency coupling between neuronal oscillations. Trends Cogn Sci 11(7):267-269

24. Paxinos G, Watson C (2006) The rat brain in stereotaxic coordinates: hard, cover. Elsevier

25. Kraeuter A-K, Guest PC, Sarnyai Z (2019) The Y-maze for assessment of spatial working and reference memory in mice. Pre-clinical models. Springer, Berlin, pp 105-111

26. Pestka S, Krause CD, Walter MR (2004) Interferons, interferon-like cytokines, and their receptors. Immunol Rev 202(1):8-32

27. Tort AB, Komorowski RW, Manns JR, Kopell NJ, Eichenbaum H (2009) Theta-gamma coupling increases during the learning of item-context associations. Proc Natl Acad Sci 106(49):20942-20947

28. Salimi M, Ghazvineh S, Nazari M, Dehdar K, Garousi M, Zare M, Tabasi F, Jamaati H, Salimi A, Barkley V (2021) Allergic rhinitis impairs working memory in association with drop of hippocampal-Prefrontal coupling. Brain Res 1758:147368

29. Seth AK, Barrett AB, Barnett L (2015) Granger causality analysis in neuroscience and neuroimaging. J Neurosci 35(8):3293-3297

30. Baccalá LA, Sameshima K (2001) Partial directed coherence: a new concept in neural structure determination. Biol Cybern 84(6):463-474

31. Bressler SL, Seth AK (2011) Wiener-Granger causality: a well established methodology. Neuroimage 58(2):323-329

32. Farokhzadi M, Hossein-Zadeh G-A, Soltanian-Zadeh H (2018) Nonlinear effective connectivity measure based on adaptive neuro fuzzy inference system and granger causality. Neuroimage 181:382-394
33. Hu S, Jia X, Zhang J, Kong W, Cao Y (2015) Shortcomings/limitations of blockwise granger causality and advances of blockwise new causality. IEEE Transactions Neural Netw Learning Syst 27(12):2588-2601

34. Palaniappan R (2006) Towards optimal model order selection for autoregressive spectral analysis of mental tasks using genetic algorithm. Int J Comput Sci Netw Secur 6(1A):153-162

35. Wu M-H, Frye RE, Zouridakis G (2011) A comparison of multivariate causality based measures of effective connectivity. Comput Biol Med 41(12):1132-1141

36. Egorov AV, Draguhn A (2013) Development of coherent neuronal activity patterns in mammalian cortical networks: common principles and local hetereogeneity. Mech Dev 130 (6-8):412-423

37. Nácher V, Ledberg A, Deco G, Romo R (2013) Coherent delta-band oscillations between cortical areas correlate with decision making. Proc Natl Acad Sci 110(37):15085-15090

38. Liebe S, Hoerzer GM, Logothetis NK, Rainer G (2012) Theta coupling between $\mathrm{V} 4$ and prefrontal cortex predicts visual short-term memory performance. Nat Neurosci 15(3):456-462

39. Gray CM, König P, Engel AK, Singer W (1989) Oscillatory responses in cat visual cortex exhibit inter-columnar synchronization which reflects global stimulus properties. Nature 338(6213):334-337

40. Lisman JE, Idiart MA (1995) Storage of 7+/-2 short-term memories in oscillatory subcycles. Science 267(5203):1512-1515

41. Montgomery SM, Buzsáki G (2007) Gamma oscillations dynamically couple hippocampal CA3 and CA1 regions during memory task performance. Proc Natl Acad Sci 104(36):14495-14500

42. Tort AB, Brankačk J, Draguhn A (2018) Respiration-entrained brain rhythms are global but often overlooked. Trends Neurosci 41 (4):186-197

43. Monto S, Palva S, Voipio J, Palva JM (2008) Very slow EEG fluctuations predict the dynamics of stimulus detection and oscillation amplitudes in humans. J Neurosci 28(33):8268-8272

44. Penny W, Duzel E, Miller K, Ojemann J (2008) Testing for nested oscillation. J Neurosci Method 174(1):50-61

45. Cohen MX, Axmacher N, Lenartz D, Elger CE, Sturm V, Schlaepfer TE (2009) Good vibrations: cross-frequency coupling in the human nucleus accumbens during reward processing. J Cogn Neurosci 21(5):875-889

46. Bragin A, Jandó G, Nádasdy Z, Hetke J, Wise K, Buzsáki G (1995) Gamma $(40-100 \mathrm{~Hz}$ ) oscillation in the hippocampus of the behaving rat. J Neurosci 15(1):47-60

47. Charpak S, Pare D, Llinás R (1995) The entorhinal cortex entrains fast CA1 hippocampal oscillations in the anaesthetized guinea-pig: role of the monosynaptic component of the perforant path. Eur J Neurosci 7(7):1548-1557

48. Bressler SL, Freeman WJ (1980) Frequency analysis of olfactory system EEG in cat, rabbit, and rat. Electroencephalogr Clin Neurophysiol 50(1-2):19-24

49. Bressler SL (1988) Changes in electrical activity of rabbit olfactory bulb and cortex to conditioned odor stimulation. Behav Neurosci 102(5):740

50. Singer W (1993) Synchronization of cortical activity and its putative role in information processing and learning. Annu Rev Physiol 55(1):349-374

51. Grosmaitre X, Santarelli LC, Tan J, Luo M, Ma M (2007) Dual functions of mammalian olfactory sensory neurons as odor detectors and mechanical sensors. Nat Neurosci 10(3):348-354

52. Lockmann AL, Laplagne DA, Leão RN, Tort AB (2016) A respirationcoupled rhythm in the rat hippocampus independent of theta and slow oscillations. J Neurosci 36(19):5338-5352

53. Chi VN, Müller C, Wolfenstetter T, Yanovsky Y, Draguhn A, Tort AB, Brankačk J (2016) Hippocampal respiration-driven rhythm distinct from theta oscillations in awake mice. J Neurosci 36(1):162-177

54. Ito J, Roy S, Liu Y, Cao Y, Fletcher M, Lu L, Boughter J, Grün S, Heck D (2014) Whisker barrel cortex delta oscillations and gamma power in the awake mouse are linked to respiration. Nat Commun 5(1):1-10

55. Macrides F, Eichenbaum HB, Forbes WB (1982) Temporal relationship between sniffing and the limbic theta rhythm during odor discrimination reversal learning. J Neurosci 2(12):1705-1717

56. Kay LM (2005) Theta oscillations and sensorimotor performance. Proc Natl Acad Sci 102(10):3863-3868

57. Fujisawa S, Buzsáki G (2011) A 4 Hz oscillation adaptively synchronizes prefrontal, VTA, and hippocampal activities. Neuron 72(1):153-165 
58. Wu R, Liu Y, Wang L, Li B, Xu F (2017) Activity patterns elicited by airflow in the olfactory bulb and their possible functions. J Neurosci 37(44):10700-10711

59. Igarashi KM, Lu L, Colgin LL, Moser M-B, Moser El (2014) Coordination of entorhinal-hippocampal ensemble activity during associative learning. Nature 510(7503):143-147

60. Semba K, Komisaruk B (1984) Neural substrates of two different rhythmical vibrissal movements in the rat. Neuroscience 12(3):761-774
61. Lockmann AL, Laplagne DA, Tort AB (2018) Olfactory bulb drives respiration-coupled beta oscillations in the rat hippocampus. Eur J Neurosci 48(8):2663-2673

\section{Publisher's Note}

Springer Nature remains neutral with regard to jurisdictional claims in published maps and institutional affiliations.
Ready to submit your research? Choose BMC and benefit from:

- fast, convenient online submission

- thorough peer review by experienced researchers in your field

- rapid publication on acceptance

- support for research data, including large and complex data types

- gold Open Access which fosters wider collaboration and increased citations

- maximum visibility for your research: over $100 \mathrm{M}$ website views per year

At BMC, research is always in progress.

Learn more biomedcentral.com/submissions 